\title{
Effect of Oral Branched-Chain Amino Acids on Serum Albumin Concentration in Heart Failure Patients with Hypoalbuminemia: Results of a Preliminary Study
}

\author{
Yuichi Uchino $^{2}$ Masafumi Watanabe ${ }^{1} \cdot$ Munenori Takata $^{1} \cdot$ Eisuke Amiya $^{1}$. \\ Kensuke Tsushima $^{1}$ - Takeshi Adachi ${ }^{3}$ - Yukio Hiroi ${ }^{4}$ ' Toshikazu Funazaki ${ }^{5}$. \\ Issei Komuro ${ }^{1}$
}

Published online: 6 March 2018

(C) The Author(s) 2018. This article is an open access publication

\begin{abstract}
Background We conducted a randomized, controlled trial to determine whether supplementation with oral branched-chain amino acids (BCAAs) improves serum albumin and clinical outcomes in heart failure (HF) patients with hypoalbuminemia. Methods and results We randomly assigned 18 in-hospital $\mathrm{HF}$ patients with serum albumin $<3.5 \mathrm{~g} / \mathrm{dL}$ to receive oral BCAA granules $\left(\right.$ LIVACT $^{\circledR}$ ) for 28 days during their hospital stay or until discharge (BCAA group; $N=9$ ) or to receive no supplementation (controls; $N=9$ ), in addition to recommended HF therapy. The primary endpoints were changes from baseline in serum albumin and cardiothoracic ratio (CTR). Sixteen patients completed the study. The mean ( \pm standard deviation) period of BCAA supplementation was $18.4 \pm 8.4$ days. Serum albumin significantly increased in the BCAA group [mean difference vs baseline, $0.44 \mathrm{~g} / \mathrm{dL} ; 95 \%$ confidence interval (CI) 0.13-0.76; $P=0.014]$ and did not change in controls $(0.18 \mathrm{~g} / \mathrm{dL} ; 95 \%$ CI -0.05 to $0.40 ; P=0.108)$. CTR significantly decreased in the BCAA group $(-2.3 \%$; $95 \% \mathrm{CI}-3.8$ to
\end{abstract}

Masafumi Watanabe

masafumi-tky@umin.net

1 Department of Cardiovascular Medicine, Graduate School of Medicine, University of Tokyo, 7-3-1 Hongo, Bunkyo-ku, Tokyo 113-8655, Japan

2 Sanno Hospital, International University of Health and Welfare, Tokyo, Japan

3 Department of Cardiology, National Defense Medical College, Saitama, Japan

4 Division of Cardiology, National Center for Global Health and Medicine, Tokyo, Japan

5 Department of Cardiovascular Medicine, Saiseikai Kawaguchi General Hospital, Saitama, Japan
$-0.8 ; P=0.014)$ and did not change in controls $(-1.0 \%$; $95 \% \mathrm{CI}-2.3$ to $0.3 ; P=0.111$ ).

Conclusion In-hospital HF patients with hypoalbuminemia supplemented with BCAAs showed increased serum albumin and decreased CTR.

Clinical trial registration number UMIN000004488 [http:// www.umin.ac.jp/ctr/index.htm]

\section{Key Points}

The present study demonstrated that in-hospital heart failure patients with hypoalbuminemia showed increased serum albumin, decreased cardiothoracic ratio, and increased cholinesterase after supplementation with oral branched-chain amino acids (BCAAs).

The increase in albumin during the treatment of heart failure was finely correlated with the changes of clinical parameters such as cardiothoracic ratio and body weight.

\section{Introduction}

Heart failure (HF) has become a growing burden on healthcare systems, and hypoalbuminemia is a common complication of HF, affecting $18-52 \%$ of patients [1-5]. Possible causes include appetite loss, malnutrition, liver dysfunction, inflammatory cytokines [2], and cardiac cachexia [6]. Serum albumin is required not only to maintain intravascular colloid oncotic pressure, but also efficient delivery of loop diuresis to its target site. For these 
reasons, hypoalbuminemia exacerbates edema and congestion and is related to poor clinical outcomes in patients with acute and chronic HF [2, 3, 5, 7, 8]. Effective therapies to improve serum albumin in patients with $\mathrm{HF}$ are unknown.

Branched-chain amino acid (BCAA) supplementation, consisting of valine, leucine, and isoleucine, has already been used for patients with advanced liver cirrhosis and provides benefit by improving the serum amino acid profile and increasing albumin concentrations [9, 10]. BCAA supplementation is also beneficial for patients with cancer [11] or those undergoing hemodialysis, by improving appetite loss [12]. BCAAs also ameliorate HF with cardiac cachexia in rats [13]. However, the efficacy of BCAAs in patients with HF is unclear.

The main hypothesis in this study is that the intervention of BCAA supplementation for hypoalbuminemia might improve clinical outcomes of HF patients. To elucidate the efficacy and safety of oral BCAA supplementation for patients with HF with hypoalbuminemia, we conducted a prospective, multicenter, randomized, controlled study of in-hospital patients with HF.

\section{Methods}

\subsection{Study Design}

This was a prospective, multicenter, randomized, open-label trial. The study was conducted in accordance with the Declaration of Helsinki. The study protocol was approved by the ethics committees of each participating institution. Written informed consent was obtained from all patients. The trial was registered at http://www.umin.ac.jp/ctr/index. htm, with the identifier UMIN000004488.

\subsection{Patients}

Patients 20 years of age or older with decompensated HF who were hospitalized in participating hospitals in Japan between November 2010 and October 2014 were assessed for eligibility. HF was diagnosed by at least one subjective symptom (dyspnea or orthopnea) and one sign (leg edema, jugular vein dilatation, pulmonary rales, or pulmonary vascular congestion on chest X-ray) of fluid retention. Eligible patients were those who showed serum albumin concentrations of $<3.5 \mathrm{~g} / \mathrm{dL}$ twice during hospitalization. The exclusion criteria were determined mainly for safety. Patients with serum creatinine $>3.0 \mathrm{mg} / \mathrm{dL}$, blood urea nitrogen $>60 \mathrm{mg} / \mathrm{dL}$, nephrotic syndrome, hemoglobin A1c $>8.0 \%$, liver cirrhosis, congenital abnormalities of amino acid metabolism, severe ventricular arrhythmia, mechanical ventilation, or severe infection were excluded.
After the hospitalization due to exacerbation of HF, initial stabilization was performed until patients could take meals without difficulties, and patient recruitment was performed.

\subsection{Study Procedures}

Eligible patients were randomly assigned to the BCAA group or the control group. Randomization was carried out by the University Hospital Clinical Trial Alliance system and was stratified according to serum albumin of $2.8 \mathrm{~g} / \mathrm{dL}$ and an age of 75 years. Control group patients were treated with recommended HF therapy. BCAA group patients were orally supplemented with one pack of BCAA granules $\left(\right.$ LIVACT $^{\circledR}$, Ajinomoto Co., Tokyo, Japan) containing $1144 \mathrm{mg}$ of L-valine, $1904 \mathrm{mg}$ of L-leucine, and $952 \mathrm{mg}$ of L-isoleucine, 3 times a day for more than 7 days during their hospital stay until hospital discharge, in addition to receiving recommended HF therapy [14]. Daily food intake was mandated by the dieticians as $25-35 \mathrm{kcal} / \mathrm{kg} / \mathrm{day}$ and $1.0-1.4 \mathrm{~g}$ protein/ $\mathrm{kg} / \mathrm{day}$, and $6 \mathrm{~g}$ sodium restriction/day was also performed.

The patients were monitored for 28 days during hospital stay or until hospital discharge. Body weight, blood pressure, chest X-ray, daily food intake proportion, serum albumin, B-type natriuretic peptide (BNP), cholinesterase, blood urea nitrogen, serum creatinine, blood ammonia, and BCAA concentration were measured at the start and the end of the study. Amino acid concentrations were measured by Ajinomoto Co. Patients who were discharged or dropped out before taking BCAAs for 7 days were excluded from the analysis.

\subsection{Study Outcomes}

The primary endpoints were changes from baseline in serum albumin and cardiothoracic ratio (CTR). The secondary endpoints included BNP, BCAA concentration, Fisher ratio (total BCAA concentration divided by total concentration of phenylalanine and tryptophan), food intake proportion, and length of hospital stay. The safety endpoints were serum creatinine, blood urea nitrogen, and blood ammonia.

\subsection{Sample Size Calculation}

We calculated that a total of 34 patients were required to obtain $80 \%$ power to detect a difference in serum albumin and CTR between the two groups with a significance level of 0.05 for a two-sided test. The sample size was overestimated to be 40 to allow potential drop-out of $15 \%$ of the patients.

\subsection{Statistical Analysis}

Quantitative variables were compared between the two groups by Student's $t$ test or the Mann-Whitney $U$ test if 
appropriate for unpaired data. Changes in quantitative variables during treatment were assessed by Student's $t$ test for paired data. Categorical variables were compared by Pearson's $\chi^{2}$ test or Fisher's exact test. Single regression analysis was used as appropriate. A $P$ value of 0.05 was considered to indicate a statistically significant difference. If appropriate, Bonferroni correction was to be performed. All tests were two-sided.

\section{Results}

\subsection{Patients}

Between October 2010 and September 2014, 18 patients were enrolled and randomly assigned to the BCAA group $(N=9)$ or the control group $(N=9)$. One patient in the BCAA group wished to discontinue the study and dropped out 3 days after enrollment. Another patient in the BCAA group was discharged from the hospital before receiving
7 days of BCAA supplementation and was excluded from the analysis. Data from a total of 16 patients were analyzed. The baseline characteristics of the two groups are summarized in Table 1. No significant differences were observed between the groups in baseline characteristics. There was one patient with intravenous inotropic agents in the BCAA group, and one patient with intravenous diuretic therapy and one with human atrial natriuretic peptide in the control group at the start of BCAA.

The mean $[ \pm$ standard deviation $(\mathrm{SD})]$ period of BCAA supplementation was $18.4 \pm 8.4$ days. Two patients in the BCAA group were excluded from the CTR analysis because chest $\mathrm{X}$-rays with the patient in the same position before and after treatment were not available.

\subsection{Efficacy Results}

The results for each endpoint are listed in Table 2. Serum albumin significantly increased in the BCAA group [mean difference vs baseline, $0.44 \mathrm{~g} / \mathrm{dL} ; 95 \%$ confidence interval

Table 1 Baseline characteristics of BCAA and control groups

\begin{tabular}{|c|c|c|c|}
\hline Characteristic & $\operatorname{BCAA}(N=7)$ & Control $(N=9)$ & $P$ value \\
\hline Age, years & $72 \pm 13$ & $80 \pm 8$ & 0.17 \\
\hline Female gender, $n(\%)$ & $4(57)$ & $5(56)$ & 0.94 \\
\hline Body weight, $\mathrm{kg}$ & $54.3 \pm 12.2$ & $48.4 \pm 17.8$ & 0.45 \\
\hline Body-mass index, $\mathrm{kg} / \mathrm{m}^{2}$ & $23 \pm 4$ & $19 \pm 5$ & 0.14 \\
\hline NYHA class $(1 / 2 / 3 / 4)$ & $0 / 0 / 1 / 6$ & $0 / 0 / 5 / 4$ & 0.15 \\
\hline Systolic blood pressure, $\mathrm{mmHg}$ & $106 \pm 16$ & $111 \pm 24$ & 0.68 \\
\hline Diastolic blood pressure, $\mathrm{mmHg}$ & $58 \pm 11$ & $57 \pm 12$ & 0.96 \\
\hline Heart rate, beats/min & $75 \pm 11$ & $79 \pm 12$ & 0.52 \\
\hline Cardiothoracic ratio, $\%$ & $61.2 \pm 3.7$ & $62.2 \pm 5.6$ & 0.88 \\
\hline Left ventricular ejection fraction, $\%$ & $0.41 \pm 0.11$ & $0.42 \pm 0.14$ & 0.96 \\
\hline Left ventricular end-diastolic diameter, $\mathrm{mm}$ & $59 \pm 10$ & $50 \pm 18$ & 0.23 \\
\hline Food intake proportion, $\%$ & $87 \pm 17$ & $82 \pm 27$ & 0.69 \\
\hline Serum albumin, $\mathrm{g} / \mathrm{dL}$ & $3.0 \pm 0.3$ & $2.9 \pm 0.4$ & 0.60 \\
\hline $\mathrm{BCAA}, \mu \mathrm{mol} / \mathrm{L}$ & $332 \pm 57$ & $373 \pm 103$ & 0.36 \\
\hline Fisher ratio & $2.9 \pm 0.9$ & $2.8 \pm 0.9$ & 0.91 \\
\hline B-type natriuretic peptide, $\mathrm{pg} / \mathrm{mL}$ & $444 \pm 482$ & $463 \pm 254$ & 0.88 \\
\hline Serum creatinine, mg/dL & $0.86 \pm 0.49$ & $1.21 \pm 0.68$ & 0.24 \\
\hline Blood urea nitrogen, mg/dL & $17 \pm 7$ & $25 \pm 15$ & 0.15 \\
\hline Ammonia, $\mu \mathrm{g} / \mathrm{dL}$ & $46 \pm 14$ & $31 \pm 13$ & 0.079 \\
\hline Cholinesterase, IU/L & $191 \pm 29$ & $170 \pm 41$ & 0.25 \\
\hline ACE inhibitors or ARBs & $6(85.7 \%)$ & $6(66.7 \%)$ & 0.37 \\
\hline Beta-blockers & $4(57.1 \%)$ & $7(77.8 \%)$ & 0.15 \\
\hline Aldosterone receptor blockers & $6(85.7 \%)$ & $7(77.8 \%)$ & 0.77 \\
\hline Timing of the start of this study (days after hospitalization) & $9.7 \pm 2.9$ & $13.3 \pm 10.7$ & 0.40 \\
\hline
\end{tabular}

Data for continuous variables are presented as mean \pm standard deviation

$A C E$ angiotensin converting enzyme, $A R B$ angiotensin II receptor blocker, $B C A A$ branched-chain amino acid, NYHA New York Heart Association 
Table 2 Results of primary, secondary, exploratory and safety endpoints

\begin{tabular}{|c|c|c|c|}
\hline \multirow[t]{2}{*}{ Endpoints } & \multicolumn{2}{|c|}{ Difference vs baseline $(95 \% \mathrm{CI})$} & \multirow[t]{2}{*}{ Comparison of changes, BCAA vs control } \\
\hline & BCAA $(N=7)$ & Control $(N=9)$ & \\
\hline Hospital stay (days) & $29.1 \pm 10.2$ & $28.8 \pm 12.2$ & 0.95 \\
\hline Study duration (days) & $15.6 \pm 7.9$ & $10.1 \pm 5.4$ & 0.15 \\
\hline \multicolumn{4}{|l|}{ Primary efficacy endpoint } \\
\hline Change of serum albumin, $\mathrm{g} / \mathrm{dL}$ & $0.44(0.13 \text { to } 0.76)^{*}$ & $0.18(-0.05$ to 0.40$)$ & $P=0.117$ \\
\hline Change of cardiothoracic ratio, $\%$ & $-2.3(-3.8 \text { to }-0.8)^{\uparrow, *}$ & $-1.0(-2.3$ to 0.3$)$ & $P=0.161$ \\
\hline \multicolumn{4}{|l|}{ Secondary efficacy endpoint } \\
\hline Change of BNP, $\mathrm{pg} / \mathrm{mL}$ & $-269(-594$ to 56$)$ & $-114(-297$ to 69$)$ & $P=0.31$ \\
\hline Change of BCAA, $\mu \mathrm{mol} / \mathrm{L}$ & $73(-8$ to 154$)$ & $21(-24$ to 67$)$ & $P=0.182$ \\
\hline Change of Fisher ratio & $0.64(-0.06$ to 1.33$)$ & $0.08(-0.33$ to 0.49$)$ & $P=0.102$ \\
\hline Change of food intake proportion, $\%$ & $8(-11$ to 26$)$ & $-4(-18$ to 11$)$ & $P=0.27$ \\
\hline Hospital stay length, mean \pm SD days & $29 \pm 10$ & $29 \pm 12$ & $P=0.95$ \\
\hline \multicolumn{4}{|l|}{ Exploratory endpoint } \\
\hline Change of body weight, $\mathrm{kg}$ & $-3.0(-6.4$ to 0.5$)$ & $-1.6(-3.2$ to 0.1$)$ & $P=0.33$ \\
\hline Change of systolic BP, mmHg & $-5(-21$ to 12$)$ & $-14(-27 \text { to }-1)^{*}$ & $P=0.30$ \\
\hline Change of diastolic $\mathrm{BP}, \mathrm{mmHg}$ & $0(-14$ to 14$)$ & $-8(-19$ to 3$)$ & $P=0.32$ \\
\hline Change of cholinesterase, IU/L & $38(9 \text { to } 67)^{*}$ & $7(-16$ to 29$)$ & $P=0.059$ \\
\hline \multicolumn{4}{|l|}{ Safety endpoint } \\
\hline Change of serum creatinine, $\mathrm{mg} / \mathrm{dL}$ & $0.03(-0.07$ to 0.12$)$ & $0.04(-0.09$ to 0.17$)$ & $P=0.89$ \\
\hline Change of urea nitrogen, $\mathrm{mg} / \mathrm{dL}$ & $9.1(1.6 \text { to } 16.6)^{*}$ & $2.8(-3.3$ to 8.9$)$ & $P=0.146$ \\
\hline Change of ammonia, $\mu \mathrm{g} / \mathrm{dL}$ & $-3.4(-18.3$ to 11.4$)$ & $-1.9(-15.0$ to 11.3$)$ & $P=0.86$ \\
\hline
\end{tabular}

Parameters with significant changes are in bold

$B C A A$ branched-chain amino acid, $B N P$ B-type natriuretic peptide, $B P$ blood pressure, $C I$ confidence interval, $S D$ standard deviation $* P<0.05$ vs baseline

${ }^{\top} N=5$; two patients in BCAA group were excluded from the cardiothoracic ratio analysis because chest X-rays with the same position (anteriorposterior or posterior-anterior) before and after BCAA supplementation were not available

(CI) $0.13-0.76 ; P=0.014]$ and did not change in controls. CTR significantly decreased in the BCAA group $(-2.3 \%$; $95 \% \mathrm{CI}-3.8$ to $-0.8 ; P=0.014)$ and did not change in controls. BNP did not change in the BCAA group or the control group. BCAA concentration, Fisher ratio, and food intake proportion did not change in either group. Heart rate and the level of C-reactive protein did not change in either group. The length of hospital stay did not differ between the groups. Systolic blood pressure did not change in the BCAA group $(-5 \mathrm{mmHg}$; $95 \% \mathrm{CI}-21$ to $12 ; P=0.56$ ) and decreased in controls $(-14 \mathrm{mmHg} ; 95 \% \mathrm{CI}-27$ to $-1 ; P=0.034)$. Cholinesterase increased in the BCAA group (38 IU/L; 95\% CI 9.2-67; $P=0.018$ ) and did not change in controls. There were no differences between the two groups in changes of all the endpoints.

The correlations among changes in serum albumin, cholinesterase, body weight, and CTR for all patients are shown in Figure 1. Changes in the serum albumin level were positively correlated with changes in the cholinesterase level $(R=0.82, P<0.001)$ and negatively correlated with changes in body weight $(R=-0.87, P<0.001)$ and CTR $(R=-0.62, P=0.017)$.

\subsection{Safety Results}

Blood urea nitrogen increased in the BCAA group $(9.1 \mathrm{mg} /$ dL; 95\% CI 1.6-16.6; $P=0.025$ ) and did not change in controls. Serum creatinine and ammonia did not change in either group.

\section{Discussion}

The present study demonstrated that in-hospital HF patients with hypoalbuminemia showed increased serum albumin, decreased CTR, and increased cholinesterase, in contrast to controls. These results suggest the efficacy of supplementation with BCAAs for treatment of HF in patients with hypoalbuminemia.

Hypoalbuminemia commonly affects patients with HF [1-5] and is associated with poor clinical outcomes in patients with acute and chronic HF [2, 3, 5, 7, 8]. Among several causes, malnutrition and inflammation were factors causing hypoalbuminemia in the setting of HF. In this study, malnutrition in HF was presumed to be a target for 


\section{A}

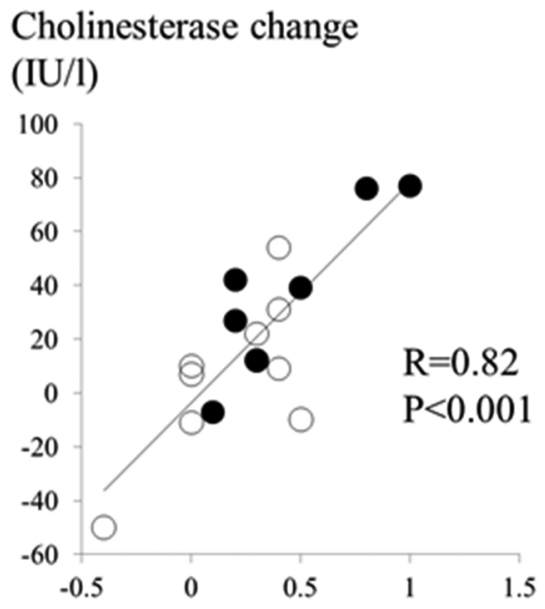

Albumin change $(\mathrm{g} / \mathrm{dl})$
B
Body weight change

$(\mathrm{kg})$
C Cardiothoracic ratio change (\%)

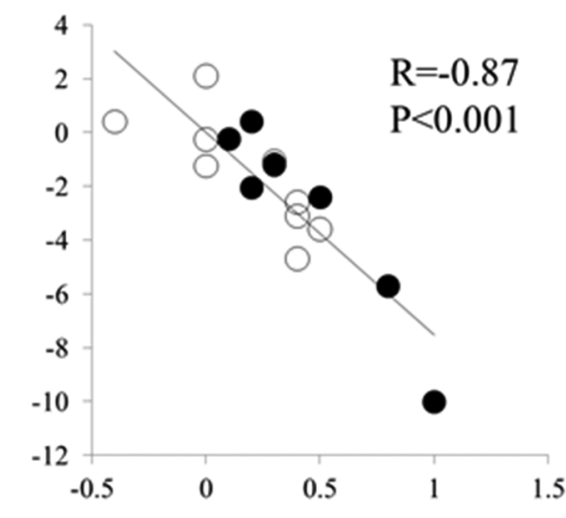

Albumin change ( $\mathrm{g} / \mathrm{dl})$

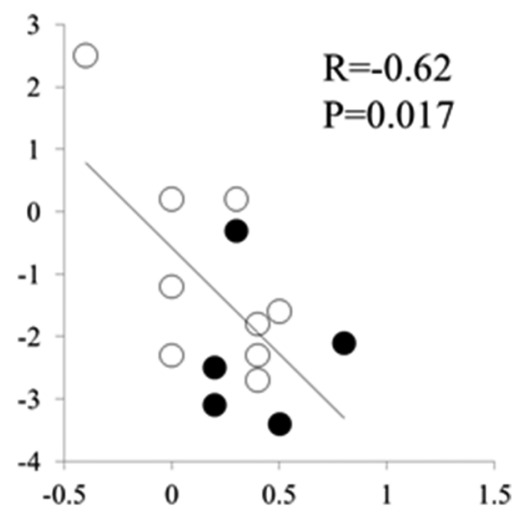

Albumin change $(\mathrm{g} / \mathrm{dl})$

\section{BCAA O controls}

Fig. 1 Correlations between serum albumin change and a cholinesterase change, $\mathbf{b}$ body weight change, and $\mathbf{c}$ cardiothoracic ratio change, respectively. Two patients in BCAA group were excluded from the cardiothoracic ratio analysis because chest $\mathrm{X}$-rays

BCAA treatment [8]. We examined the hypothesis that the intervention for hypoalbuminemia might improve clinical outcomes of $\mathrm{HF}$ patients. This is the first report to demonstrate that BCAA supplementation in HF patients could improve serum albumin levels, serum cholinesterase levels, and CTR. Furthermore, we found no major complications, such as hypotension, or increases in serum creatinine, except a slight elevation of blood urea nitrogen levels.

In this study, we used CTR as an evaluation for the status of HF. There was a manuscript that demonstrated the correlative relationship between CTR and the level of BNP [15]. However, CTR has a distinctive feature that CTR reflects right- rather than left-sided heart size in patients with HF [16]. Indeed, right $\mathrm{HF}$ has a closer association with malnutrition than left-sided HF [17, 18]. Therefore, CTR is presumed to be more appropriate for the evaluation of HF from the viewpoint of malnutrition.

As shown in Figure 1, increases in albumin were correlated with decreases in CTR and body weight. These results indicate that BCAAs ameliorated fluid retention in patients with congestive HF probably through an increase in serum albumin. We found no significant difference in systolic blood pressure between the BCAA group and controls. This finding may indicate the safety of BCAA supplementation in the population with low blood pressure, with maintenance of intravascular osmolality and volume via improved serum albumin levels, even with restricted sodium intake and the use of diuretics [19]. with the same position (anterior-posterior or posterior-anterior) before and after BCAA supplementation were not available. $B C A A$ branched-chain amino acid

One possible explanation for this result is that BCAAs serve as substrates of protein synthesis. BCAAs, particularly leucine, activate the mammalian target of rapamycin (mTOR) [20], promoting albumin synthesis [9]. The correlative relationship between albumin and cholinesterase supports the beneficial effect of BCAAs on liver protein synthesis in this study population. However, there might be a possibility that the improvement of CTR might be derived from an efficient decongestion induced by BCAA administration.

There are several limitations to the present study. First, the target sample size was initially 40 , but only 18 patients were enrolled. The study may have had insufficient power to detect differences in the endpoints between the groups. Indeed, we could not exhibit a statistically significant superior response in serum albumin level with BCAA supplementation. Second, optimal doses of BCAAs were not evaluated. The patients were only monitored for 28 days or until discharge from the hospital. The study was stopped at the time of discharge, and the SD value of study duration was variously distributed. Because hemoconcentration may possibly affect the level of albumin, more stable patients with HF would be suitable for an investigation into the efficacy of BCAAs for improvement of hypoalbuminemia in the treatment of HF. Future studies should evaluate the long-term clinical outcomes, including those in outpatients, especially whether BCAAs decrease repeated admissions. Furthermore, the assessment of sarcopenia might elucidate the mechanistic insight of BCAA treatment in $\mathrm{HF}$. 
In conclusion, in-hospital HF patients with hypoalbuminemia showed increased serum albumin and decreased CTR after supplementation with BCAAs. Our results indicate the efficacy of BCAA supplementation for $\mathrm{HF}$ patients with hypoalbuminemia.

Acknowledgements The authors thank Dr. Kenji Takehana (Ajinomoto Pharmaceuticals Co. Ltd.) for discussions on amino acid metabolism. The authors also thank Ajinomoto Co. Inc. for their help with amino acid analysis.

\section{Compliance with Ethical Standards}

Funding This work was funded by a grant from Ajinomoto Pharmaceuticals Co. Ltd., to Dr. Masafumi Watanabe.

Conflict of interest Yuichi Uchino, Masafumi Watanabe, Munenori Takata, Eisuke Amiya, Kensuke Tsushima, Takeshi Adachi, Yukio Hiroi, Toshikazu Funazaki, and Issei Komuro have no conflicts of interest that might be relevant to this work.

Open Access This article is distributed under the terms of the Creative Commons Attribution-NonCommercial 4.0 International License (http://creativecommons.org/licenses/by-nc/4.0/), which permits any noncommercial use, distribution, and reproduction in any medium, provided you give appropriate credit to the original author(s) and the source, provide a link to the Creative Commons license, and indicate if changes were made.

\section{References}

1. Allen LA, Felker GM, Pocock S, McMurray JJ, Pfeffer MA, Swedberg K, et al. Liver function abnormalities and outcome in patients with chronic heart failure: data from the Candesartan in Heart Failure: Assessment of Reduction in Mortality and Morbidity (CHARM) program. Eur J Heart Fail. 2009;11:170-7.

2. Horwich TB, Kalantar-Zadeh K, MacLellan RW, Fonarow GC. Albumin levels predict survival in patients with systolic heart failure. Am Heart J. 2008;155:883-9.

3. Novack V, Pencina M, Zahger D, Fuchs L, Nevzorov R, Jotkowitz A, et al. Routine laboratory results and thirty day and one-year mortality risk following hospitalization with acute decompensated heart failure. PLoS One. 2010;5:e12184.

4. Lau GT, Tan HC, Kritharides L. Type of liver dysfunction in heart failure and its relation to the severity of tricuspid regurgitation. Am J Cardiol. 2002;90:1405-9.

5. Uthamalingam S, Kandala J, Daley M, Patvardhan E, Capodilupo R, Moore SA, et al. Serum albumin and mortality in acutely decompensated heart failure. Am Heart J. 2010;160:1149-55.
6. Anker SD, Coats AJ. Cardiac cachexia: a syndrome with impaired survival and immune and neuroendocrine activation. Chest. 1999;115:836-47.

7. Arques S, Ambrosi P. Human serum albumin in the clinical syndrome of heart failure. J Card Fail. 2011;17:451-8.

8. Bonilla-Palomas JL, Gámez-López AL, Moreno-Conde M, López-Ibáñez MC, Anguita-Sánchez M, de la Sacristana ÁG, et al. Hypoalbuminemia in acute heart failure patients: causes and its impact on hospital and long-term mortality. J Card Fail. 2014;20:350-8.

9. Ijichi C, Matsumura T, Tsuji T, Eto Y. Branched-chain amino acids promote albumin synthesis in rat primary hepatocytes through the mTOR signal transduction system. Biochem Biophys Res Commun. 2003;303:59-64.

10. Tajiri K, Shimizu Y. Branched-chain amino acids in liver diseases. World J Gastroenterol. 2013;19:7620-9.

11. Cangiano C, Laviano A, Meguid MM, Mulieri M, Conversano L, Preziosa I, et al. Effects of administration of oral branched-chain amino acids on anorexia and caloric intake in cancer patients. J Natl Cancer Inst. 1996;88:550-2.

12. Hiroshige K, Sonta T, Suda T, Kanegae K, Ohtani A. Oral supplementation of branched-chain amino acid improves nutritional status in elderly patients on chronic haemodialysis. Nephrol Dial Transplant. 2001;16:1856-62.

13. Tanada Y, Shioi T, Kato T, Kawamoto A, Okuda J, Kimura T. Branched-chain amino acids ameliorate heart failure with cardiac cachexia in rats. Life Sci. 2015;137:20-7.

14. Muto Y, Sato S, Watanabe A, Moriwaki H, Suzuki K, Kato A, Long-Term Survival Study Group, et al. Effects of oral branchedchain amino acid granules on event-free survival in patients with liver cirrhosis. Clin Gastroenterol Hepatol. 2005;3:705-13.

15. Ishizaka Y, Yamamoto Y, Fukunaga T, Yokota N, Kida O, Kitamura K. Plasma concentration of human brain natriuretic peptide in patients on hemodialysis. Am J Kidney Dis. 1994;24:461-72.

16. Fukuta H, Ohte N, Brucks S, Carr JJ, Little WC. Contribution of right-sided heart enlargement to cardiomegaly on chest roentgenogram in diastolic and systolic heart failure. Am J Cardiol. 2007;99:62-7.

17. Carr JG, Stevenson LW, Walden JA, Heber D. Prevalence and hemodynamic correlates of malnutrition in severe congestive heart failure secondary to ischemic or idiopathic dilated cardiomyopathy. Am J Cardiol. 1989;63:709-13.

18. Dimopoulos K, Giannakoulas G, Bendayan I, Liodakis E, Petraco R, Diller GP. Cardiothoracic ratio from postero-anterior chest radiographs: a simple, reproducible and independent marker of disease severity and outcome in adults with congenital heart disease. Int J Cardiol. 2013;166:453-7.

19. Lee DS, Ghosh N, Floras JS, Newton GE, Austin PC, Wang X, et al. Association of blood pressure at hospital discharge with mortality in patients diagnosed with heart failure. Circ Heart Fail. 2009;2:616-23.

20. Avruch J, Long X, Ortiz-Vega S, Rapley J, Papageorgiou A, Dai N. Amino acid regulation of TOR complex 1. Am J Physiol Endocrinol Metab. 2009;296:E592-602. 